\title{
Lactate Dehydrogenase and Glutamic Oxalic Transaminase Isozymes in Heart Muscle of Forensic Pathological Cases
}

\author{
Tsuneo Suzuki, Seitchi Kashimura and Kazuo Umetsu \\ Department of Forensic Medicine, Yamagata University School \\ of Medicine, Yamagata
}

\begin{abstract}
Suzuki, T., Kashimura, S. and Umetsu, K. Lactate Dehydrogenase and Glutamic Oxalic Transaminase Isozymes in Heart Muscle of Forensic Pathological Cases. Tohoku J. exp. Med., 1978, 124 (3), 267-275- LDH and GOT isozyme patterns in the heart muscle of forensic pathological cases were investigated to find out the relationship between their changes and the causes of death. In most cases of death by violence, natural causes, poisoning and cold, the sum of LDH-1 and LDH-2 averaged about $80-90 \%$ of the total LDH, and GOTs was equal to or slightly lower than GOTm. In about two thirds of cases of asphyxia and drowning, LDH-1 and LDH-2 decreased and LDH-3, LDH -4 and $\mathrm{LDH}-5$ increased. The activity of GOTs decreased and the ratio of GOTs/GOTm diminished. In neonate, $\mathrm{LDH}-3$ showed the highest activity, followed by LDH-2 and LDH-1. GOTm was much less than GOTs. In most eases of cardiac death from unknown cause and cot death, LDH-3 increased remarkably, though it was less than LDH-1 and LDH-2, and GOTm became much less than GOTs. These findings revealed that there were some similarities in the LDH and GOT isozyme patterns in the heart muscle of the cardiac death from unknown cause and cot death and that of neonate. LDH isozyme; GOT isozyme; cardiac death from unknown cause; cot death
\end{abstract}

In the heart muscle, there are many kinds of enzymes related to glycolysis or other metabolic pathways. The activities of some of these enzymes are said to be reduced or absent in dead or ischemically damaged cells. They vary greatly both in sensitivity to cellular damage and in resistance to postmortem changes. Among these enzymes, lactic dehydrogenase ( $\mathrm{LDH})$, malic dehydrogenase $(\mathrm{MDH})$, succinic dehydrogenase (SDH), glutamic oxalic transaminase (GOT), aldolase and hydroxy butylic dehydrogenase (HBD) have been investigated in relation to cardiac death (Funao and Sugimoto 1960; Aoki 1969). A decrease of the activities of these enzymes was noted in cases of acute cardiac death with pathological changes. However, the characteristic changes of these enzyme activities have not yet been elucidated in cases of cardiac death from unknown cause such as the so-called "cardiac failure of unknown etiology" (Sugai 1959) or "cardiac death with negative autopsy" (Marek 1972).

In addition to the quantitative studies of activities of these enzymes, the investigation of their qualitative changes such as alteration of isozyme patterns in

Received for publication, August 29, 1977. 
relation to various causes of death including cardiac death seems to be a clue to find some changes in the heart of the case of cardiac death from unknown cause. Among these enzymes, LDH and GOT have been known to consist of clearly defined isozymes which change their patterns according to the condition of tissue metabolism. The present study was undertaken to analyse $\mathrm{LDH}$ and GOT of the heart muscle in the forensic pathological cases of death from various causes to investigate the relationship between the changes of isozyme patterns in the heart muscle and the causes of death.

\section{Materials and Methods}

The study involved the hearts from 144 forensic pathological cases, including males, females and neonates. The death causes of these cases and the number except 10 neonates are shown in Table 1. The heart tissue extracts were prepared as follows: From the apical portion of the left ventricular wall, $0.5 \mathrm{~g}$ of the heart muscle was taken and minced, then washed with normal saline repeatedly to remove blood and ground in a homogenizer with $2 \mathrm{ml}$ of phosphate buffered saline $\left(\mathrm{pH} \mathrm{7.4)}\right.$ for $5 \mathrm{~min}$ at $0^{\circ} \mathrm{C}$. The homogenate was centrifuged at $15,000 \times \mathrm{g}$ for $60 \mathrm{~min}$ and the clear supernatant was used for electrophoretic analysis.

TABLE 1. Death causes and numbers of the cases involved in the present study

\begin{tabular}{lc}
\hline \multicolumn{1}{c}{ Death cause } & Number of cases \\
\hline Violent death & 42 \\
Natural death & 24 \\
Poisoning & 22 \\
Cold & 6 \\
Asphyxia \& Drowning & 21 \\
Cardiac death from & 12 \\
unknown cause & \\
Cot death & 7 \\
\multicolumn{2}{c}{ Total } \\
\hline
\end{tabular}

Electrophoretic separation of the isozyme of lactate dehydrogenase was performed on the agar-gel plate. The agar-gel plates were made by pouring $1 \%$ Difco Speical Noble Agar dissolved in Tris barbital buffer $(0.15 \mathrm{M}, \mathrm{pH} 8.4)$ on the $9.5 \times 8.5 \mathrm{~cm}$ glass plates to $1 \mathrm{~mm}$ thick. In the agar-gel electrophoretic analysis, 2 to $3 \mu \mathrm{l}$ of the heart extract were applied to a $4 \mathrm{~mm}$ slit made by inserting a filter paper $4 \mathrm{~mm}$ wide and $10 \mathrm{~mm}$ long. Electrophoresis was carried out for $1.5 \mathrm{hr}$ at 70 volts in $0.15 \mathrm{M}$ Tris barbital buffer ( $\mathrm{pH}$ 8.4). Then, the gel plate was covered with agar-gel containing the incubation medium for staining $\mathrm{LDH}$ and incubated at $37^{\circ} \mathrm{C}$ for $1 \mathrm{hr}$. The incubation medium was prepared freshly just before use by dissolving $0.15 \mathrm{~g}$ of Difco Special Noble Agar in $7 \mathrm{ml}$ of Tris-HCl buffer with heat according to Myers and Van Remortel (1968). After cooling to about $60^{\circ} \mathrm{C}, 0.2 \mathrm{ml}$ of sodium lactate solution ( $60 \%$ syrup) was added and mixed by swirling. Then, $2 \mathrm{ml}$ of tetrazolium solution (prepared freshly before use, MTT $1 \mathrm{mg} / \mathrm{ml}$ distilled water), $0.1 \mathrm{ml}$ of phenazine methosulfate solution (prepared just before use, $1 \mathrm{mg} / \mathrm{ml}$ distilled water) and $10 \mathrm{mg}$ of nicotinamide-adenin-dinucleotide were added and mixed by gentle swirling. After incubation with staining solution, the gel was used for densitometric determination of LDH isozyme pattern.

Electrophoretic analysis of GOT isozymes from the heart tissue extracts was carried out by starch-gel electrophoresis (Boyde and Latner 1962). The starch-gel plates were 
prepared by pouring $12 \%$ starch dissolved in Tris citrate buffer $(0.01 \mathrm{M}, \mathrm{pH} 7.5)$ on the $14 \times 14 \mathrm{~cm}$ glass plate to $1.8 \mathrm{~mm}$ thick. The samples were inserted into the thin layer of starch-gel with Toyo No. 525 filter paper, $8 \mathrm{~mm}$ wide and $1.5 \mathrm{~mm}$ long. Electrophoresis was carried out for $2 \mathrm{hr}$ at $4^{\circ} \mathrm{C}$ at about 100 volts in $0.1 \mathrm{M}$ Tris citrate buffer, $\mathrm{pH}$ 7.5. Then, the filter paper impregnated with staining solution was laid on the gel plate and incubated at $37^{\circ} \mathrm{C}$ for $5-10 \mathrm{~min}$ for demonstration of GOT isozymes. The staining mixture was made by dissolving $0.5 \mathrm{ml}$ of $0.2 \mathrm{M}$ aspartic acid, $0.2 \mathrm{ml}$ of $0.1 \mathrm{M} \alpha$-ketoglutaric acid, $50 \mu \mathrm{l}$ of $\mathrm{MDH}\left(10 \mathrm{U} / \mathrm{ml}\right.$ ) and $2 \mathrm{mg}$ of $\mathrm{NADH}_{2}$ in $2 \mathrm{ml}$ of the phosphate buffer (pH 7.4) according to Karmen et al. (1955). The gel plates were inspected under ultraviolet light and GOT activity was demonstrated on the gel as two bands of defluorescence, representing the sites of $\mathrm{NADH}_{2}$ oxidation to NAD. For assay of GOTs and GOTm, they were separated by the same electrophoretic method, and $0.8 \mathrm{~cm}$ square of gel blocks were taken at the sites of GOTs and GOTm isozyme. Then, they were extracted with $2.5 \mathrm{ml}$ of phosphate buffer, pH 7.4. Two ml of extracts from each blocks were added with $0.2 \mathrm{ml}$ of staining solution and the activities of these isozymes were measured at the wave length of $340 \mathrm{~nm}$ on the spectrophotometer.

\section{Results}

\section{$L D H$ isozyme patterns}

$\mathrm{LDH}$ consists of five different isozymes, which are composed of two subunit peptides $\mathrm{H}$ and $\mathrm{M}$, and the enzymes from $\mathrm{LDH}-1$ to $\mathrm{LDH}-5$ are able to be written HHHH, HHHM, HHMM, HMMM and MMMM, respectively (Vesell and Bearn 1961; Wróblewski and Gregory 1961). LDH-1 is nearest to the anode and $\mathrm{LDH}-5$ is nearest to the cathode.

No significant difference was observed in these patterns among any portions of the heart, any ages except neonate and any periods after death if they were taken within 2 to 3 days after death. However, some differences in LDH isozyme patterns were found among a few causes of death and neonate. The characteristic LDH zymograms and their densitometric patterns in cases of violent death, asphyxia, cardiac death from unknown cause, cot death, neonate and sepsis are shown in Fig. 1. The average percentage distribution of $\mathrm{LDH}$ isozyme fractions in the heart muscle in cases of the individual causes of death and neonates are shown in Table 2 and Fig. 2. In cases of violent death, death from cold, and death from poisoning, the sum of LDH-1 and LDH-2 averaged about $80-90 \%$ of the total LDH, and the amount of LDH-1 was a little more than or almost equal to that of $\mathrm{LDH}-2$. The activities of $\mathrm{LDH}-3, \mathrm{LDH}-4$ and $\mathrm{LDH}-5$ were much less than those of LDH-1 and LDH-2, and they occupied about only $10-5 \%, 6-3 \%$ and $3-1 \%$, respectively. On the other hand, in the heart of neonate, the amount of $\mathrm{LDH}-3$ was the highest, $29-37 \%$ of the total LDH, followed by LDH-2 $(26-33 \%)$ and LDH-1 (21-29\%). However, this characteristic pattern of LDH could not be seen in cases of 3 and 5 months after birth.

In cases of asphyxia and drowning, the average distribution of the cardiac LDH, isozymes showed more M subunit, and LDH-2 was equal to or a little higher than LDH-1 as shown in Table 2 and Fig. 2. However, in about one third of these cases, LDH-1 and LDH-2 showed marked decrease to about $30 \%$, and LDH-3, LDH-4 and LDH-5 were about 20,13 and $8 \%$, respectively. In another one third of these 


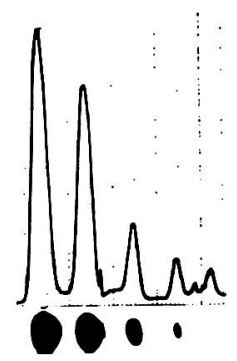

(1)

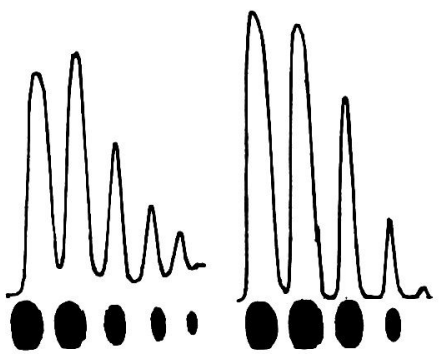

(2)
(3)

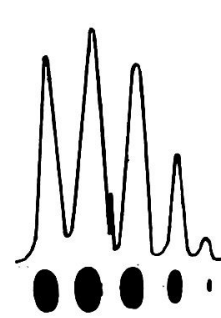

(4)

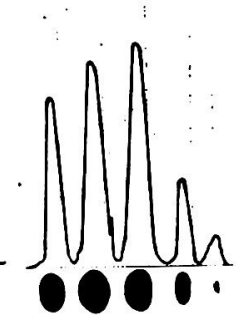

(5)

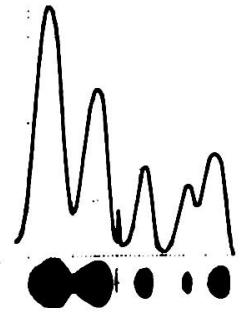

(6)

Fig. 1. Characteristic LDH zymograms and their densitometric patterns of the hearts in cases of violent death (1), asphyxia (2), cardiac death from unknown cause (3), cot death (4), neonate (5) and sepsis (6).

TABLE 2. Percentage distribution of $L D H$ isozyme fractions in the heart muscle of forensic pathological cases

\begin{tabular}{|c|c|c|c|c|c|}
\hline Death cause & LDH-1 & LDH-2 & $\mathrm{LDH} \cdot 3$ & LDH-4 & LDH-5 \\
\hline Violent death & $43.4 \pm 4.7$ & $41.5 \pm 4.1$ & $7.9 \pm 2.6$ & $4.6 \pm 2.0$ & $2.6 \pm 0.6$ \\
\hline Natural death & $43.7 \pm 4.9$ & $42.3 \pm 4.5$ & $6.6 \pm 2.8$ & $5.3 \pm 2.1$ & $2.1 \pm 0.5$ \\
\hline Poisoning & $42.8 \pm 4.3$ & $41.6 \pm 4.8$ & $8.2 \pm 3.1$ & $4.3 \pm 2.6$ & $3.1 \pm 1.3$ \\
\hline Cold & $42.3 \pm 3.9$ & $40.9 \pm 4.3$ & $7.6 \pm 2.1$ & $5.4 \pm 1.9$ & $3.8 \pm 1.5$ \\
\hline Asphyxia & $37.2 \pm 4.2$ & $37.9 \pm 4.6$ & $12.2 \pm 3.1$ & $8.3 \pm 2.1$ & $5.4 \pm 1.5$ \\
\hline $\begin{array}{l}\text { Cardiac death from } \\
\text { unknown cause }\end{array}$ & $34.3 \pm 4.5$ & $30.1 \pm 4.3$ & $25.0 \pm 2.8$ & $7.7 \pm 2.3$ & $2.9 \pm 1.3$ \\
\hline Cot death & $26.2 \pm 4.4$ & $31.9 \pm 4.2$ & $24.7 \pm 3.8$ & $13.1 \pm 2.0$ & $4.1 \pm 1.6$ \\
\hline Neonate & $25.1 \pm 3.4$ & $29.7 \pm 3.5$ & $33.2 \pm 4.5$ & $9.1 \pm 2.1$ & $2.9 \pm 1.8$ \\
\hline
\end{tabular}

cases the isozyme pattern was almost the same as in cases of violent death.

In cases of cardiac death from unknown cause, LDH-1 and LDH-2 decreased to about $39-30 \%$ and $34-26 \%$, respectively. On the other hand, LDH-3 showed an increase to about $28-23 \%$, and $\mathrm{LDH}-4$ and $\mathrm{LDH}-5$ also increased to $10-5 \%$ and $4-2 \%$, respectively. In cases of cot death, $\mathrm{LDH}-2$ was the highest, showing $36-28 \%$, followed by LDH-1 (31-22\%) and LDH-3 (28-22\%). LDH-4 and LDH-5 were $15-11 \%$ and $6-3 \%$, respectively. In cases of unexpected death due to natural causes, the average of LDH isozyme patterns was almost the same as that in the violent death cases. In these cases, however, the characteristic pattern was observed in a case of severe lung fibrosis and three cases of sepsis. In the case 


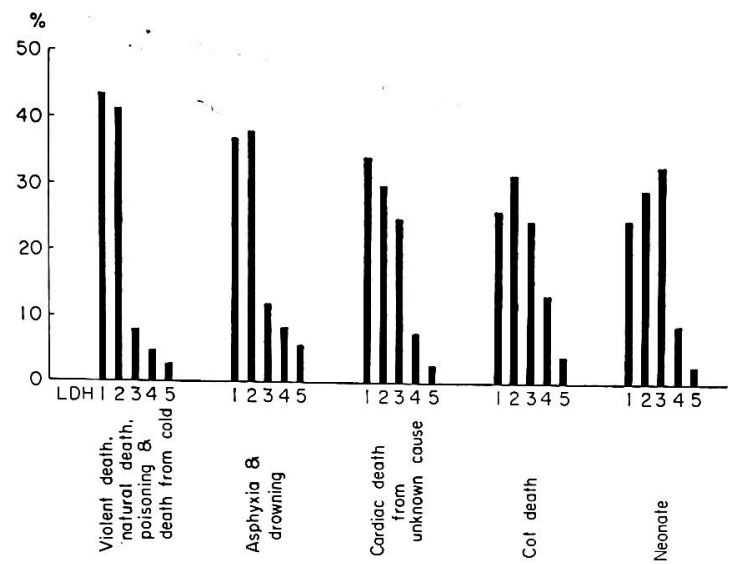

Fig. 2. Average LDH isozyme distributions in the heart of forensic pathological cases.

of lung fibrosis, LDH isozyme pattern showed more cathodic isozyme as in the asphyxia, but LDH-1 was slightly higher than LDH-2. In cases of sepsis, the most characteristic feature was a marked increase in LDH-5 and decreases in LDH1 and LDH-2. LDH-5 was about $20-15 \%$. On the other hand, LDH-1 and LDH -2 were $35-30 \%$ and $30-25 \%$, respectively.

\section{GOT isozyme patterns}

GOT consists of two different isozymes, that is, supernatant GOT (GOTs) and mitochondrial GOT (GOTm). The former migrates to the anode side, and the latter to the cathode side. No difference was found in the patterns among any portions of the heart, any ages except neonate and any periods after death if they were examined within 2 to 3 days after death. However, they showed some differences among a few causes of death and neonates. Fig. 3 shows the characteristic GOT isozyme patterns of the heart muscle in cases of violent death, asphyxia, cardiac death from unknown cause, cot death, neonate and lung fibrosis. The ratios of GOTs/GOTm in all cases examined are lumped together with individual death causes in Fig. 4. In most cases of violent death, natural death, poisoning and

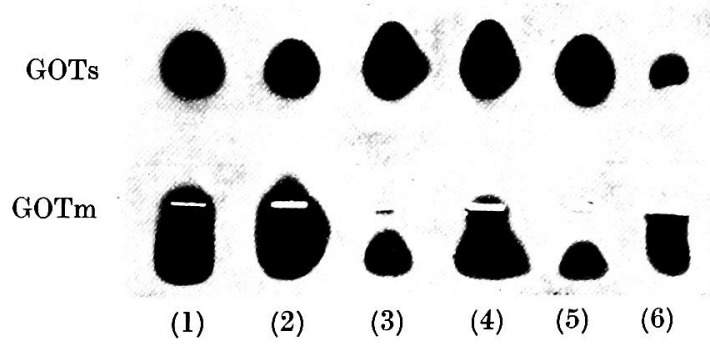

Fig. 3. Characteristic GOT isozyme patterns of the hearts in cases of violent death (1), asphyxia (2), cardiac death from unknown cause (3), cot death (4), neonate (5) and lung fibrosis (6). 


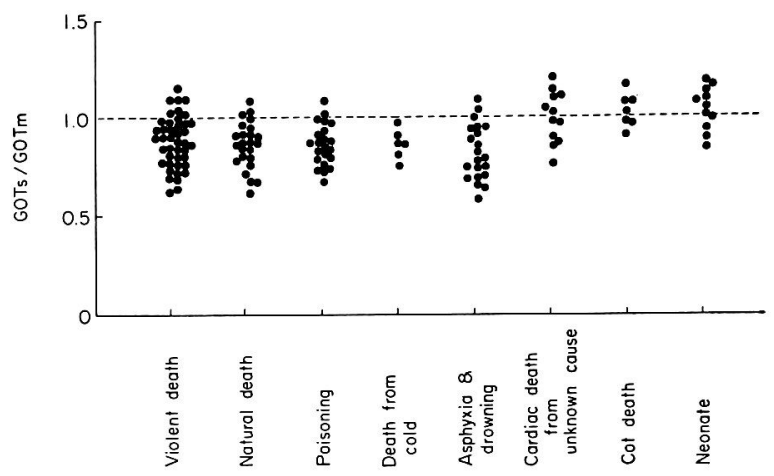

Fig. 4. Ratios of GOTs/GOTm in the heart muscle in forensic pathological cases.

death by cold, the activity of GOTm was equal to or slightly stronger than that of GOTs, and the ratio of GOTs/GOTm was 1.0-0.8. In most of neonates, GOTs showed a marked increase, and the ratio of GOTs/GOTm was 1.2-1.0. In more than half cases of asphyxia and drowning, there was a diminution of GOTs/GOTm ratio (below 0.8). In most cases of unexpected death due to natural causes, the ratio of GOTs/GOTm was almost the same as in cases of violent death. However, in the case of lung fibrosis, GOTs decreased considerably and the ratio GOTs/GOTm was 0.65 , and also in two cases of sepsis the ratio GOTs/GOTm decreased slightly to below 0.8. In 6 out of 12 cases of cardiac death from unknown cause and 4 out of 7 cases of cot death, the activity of GOTm decreased significantly and the ratio GOTs/GOTm was 1.25-1.0.

\section{Discussion}

The percentage distribution of $\mathrm{LDH}$ isozymes of human heart has been reported by various investigators (Vesell and Bearn 1961; Wróblewski and Gregory 1961; Wieme and Van Maercke 1961; Plagemann et al. 1963; Plummer et al. 1963). Their data are summarized in Table 3. Though the distribution of LDH isozymes is somewhat different among the investigators, it is clear that the percentage of $\mathrm{LDH}$ isozymes of human heart has a similar pattern. That is, the percentages of LDH1 and LDH-2 are remarkably high in comparison with those of LDH-3, LDH-4

TABLE 3. Percentage distribution of $L D H$ isozyme fraction in human heart

\begin{tabular}{lccccc}
\hline \multicolumn{1}{c}{ Reporter } & LDH-1 & LDH-2 & LDH-3 & LDH-4 & LDH-5 \\
\hline $\begin{array}{l}\text { Vesell \& Bearn (1961) } \\
\text { Wróblewski \& Gregory }\end{array}$ & $\mathbf{7 1}$ & 27 & 2 & - & - \\
$\begin{array}{c}\text { (1961) } \\
\text { Wieme \& Van }\end{array}$ & 35 & 36 & 12 & 16 & 11 \\
$\begin{array}{c}\text { Maercke (1961) } \\
\text { Plagemann et al. }\end{array}$ & 67 & 29 & 4 & $<1$ & $<1$ \\
$\begin{array}{l}\text { Pla63) } \\
\text { Plummer et al. (1963) }\end{array}$ & 53 & 29 & 16 & 2 & $<1$ \\
\hline
\end{tabular}


and LDH-5. It is said that tissues such as the heart, kidney cortex and brain, in which the metabolism is mostly aerobic, have mainly LDH-1 and LDH-2, which are chiefly composed of $\mathrm{H}$ subunit. On the other hand, tissues such as white skeletal muscle or uterus, in which metabolism is mostly anerobic, contain mainly LDH 4 and LDH-5, which are chiefly composed of M subunit. The synthesis of peptides $\mathrm{H}$ and $\mathrm{M}$ is controlled by two different genes and the activity of these genes determine the amount of each isozyme in the cell (Shaw and Barto 1963).

The percentage composition of GOT isozyme of human heart was reported by Fleisher et al. (1960). According to them, GOTs was almost equal to or slightly lower than GOTm, and the ratio GOTs/GOTm was 1.0 or slightly less than 1.0. GOT catalyzes the transfer of amino groups between aspartic acid and $\alpha$-ketoglutaric acid and is involved in amino acid and sugar metabolism. GOTs and GOTm occupy the different sites within the cell and have different biochemical and physical properties (Boyd and Grevilie 1961; Boyd 1961).

In the present study, LDH isozyme distribution in death cases from violence, natural cause, poisoning and cold had the similar pattern to those described in Table 3, and the ratio of GOTs/GOTm was $1.0-0.8$.

In about two thirds of the cases of asphyxia and drowning, the activity of LDH1 and LDH-2 in the heart diminished and that of LDH-3, LDH-4 and LDH-5 was elevated. The isozyme distribution tended to show a pattern with more M subunit. Lindy and Lajasalmi (1966) revealed that incubation of chick embryos in hypoxic environment caused an increase of subunit M in the tissue LDH, whereas incubation in aerobic condition caused a decrease of subunit M, but variation of ambient oxygen tension did not change the total $\mathrm{LDH}$ activity. Chan et al. (1962) also showed that tissue culture of chick embryonic breast muscle and heart in vitro caused a rapid synthesis of subunit $M$, and incubation of the tissue culture in $95 \% \mathrm{O}_{2}$ caused a delay in this phenomenon. They interpreted this phenomenon as an effect of oxygen on the gene directing synthesis of subunit M. Lindy et al. (1972) observed that M subunit of $\mathrm{LDH}$ isozymes increased in the rabbit aortic wall injured by pulling an inflated balloon catheter, and considered the increase in the subunit $\mathrm{M}$ percent of $\mathrm{LDH}$ as a sign of hypoxic condition in the arterial wall. These experiments suggest that the change in $\mathrm{LDH}$ isozymes composed of subunit $M$ are better suited for the formation of lactate from pyruvate in the hypoxic condition. Therefore, it seems that the increase of subunit $\mathrm{M}$ in $\mathrm{LDH}$ isozyme in the heart of the cases of asphyxia and drowning is caused by anoxia or hypoxia of the heart muscle. The changes of $\mathrm{LDH}$ isozyme patterns seem to become more characteristic with the duration of anoxic condition. According to Sawaguchi (1973), in the acutely suffocated rabbits, there was no significant change of LDH isozyme pattern in the heart muscle but marked alteration occurred in the liver. In the present study, the asphyxic or drowning cases which did not show any change in LDH isozyme pattern in the heart muscle seemed to die after such a short period of exposure to anoxic condition that no significant increase of subunit M took place. A more prominent increase of subunit $M$ was observed in the cases 
of lung fibrosis and sepsis. This is considered to be due to extremely long period of hypoxic condition of the heart muscle.

In GOT isozyme pattern in the heart, the activity of GOTs in most cases of asphyxia and drowning showed a decrease and the ratio GOTs/GOTm diminished. Fleisher et al. (1960) observed that GOTs/GOTm ratio of the heart muscle diminished as a consequence of myocardial infarction or acute suffocation. The death of myocardial cells increases the permeability of the cell membrane allowing GOT to pass out of the cell into the serum (Shikata et al. 1970). According to Katunuma et al. (1962 a, 1962 b), GOT diffusing into the serum in cases of myocardial infarction is mostly GOTs because of its lower molecularity. From these works, the decrease of GOTs in the heart muscle in asphyxia or drowning seems to be caused by its diffusion into the serum due to increasing permeability of cell membrane under hypoxic condition. The marked diminution of GOTs/GOTm ratio in cases of lung fibrosis and sepsis also seems to be caused by the long period of hypoxic condition of the heart.

In cases of neonate, the isozyme patterns of LDH and GOT were somewhat different from those in most cases of violent and asphyxic death. In LDH isozyme patterns, LDH-3 showed the highest activity, followed by $\mathrm{LDH}-2$ and LDH-1. In GOT isozyme pattern, GOTm activity decreased slightly and the ratio GOTs/GOTm increased. These characteristic isozyme patterns in the heart muscle of neonates were not observed in cases of 2 and 5 months after birth. From this fact, the characteristic isozyme pattern in neonate seems to change to the most common pattern such as in cases of violent death at an early stage after birth.

In cases of cardiac death from unknown cause and cot death, the LDH isozyme patterns showed an increase of LDH-3 with a slight elevation of $\mathrm{LDH}-4$ and $\mathrm{LDH}-$ 5 , but LDH-3 was less than LDH-1 and LDH-2. The difference of the pattern between cardiac death from unknown cause and cot death was that in the former LDH-1 was disposed to be higher than LDH-2. In the GOT isozyme distribution in these cases, the characteristic change was a decrease of GOTm, and in most cases the ratio GOTs/GOTm was 1.25-1.0. These findings revealed that there were some similarities in the LDH and GOT isozyme patterns in the heart muscle of the cases of cardiac death from unknown cause and cot death and that of the neonate. These similarities seem to have some relations to the cause of sudden death in cases of cardiac death from unknown cause and cot death.

\section{Acknowledgment}

We express our grateful thanks to Tokyo-To Medical Examiner's Office for providing samples of the heart of some sudden unexpected death cases including the cases of cardiac death from unknown cause.

\section{References}

1) Aoki, T. (1969) Quantitative changes of chemical constituents in blood and several organs after death due to various causes. In: Sudden death from internal etiology, 
edited by T. Matsukura Jap. J. legal Med., 23, 42I-449. (Japanese)

2) Boyd, J.W. (1961) The intracellular distribution, latency and electrophoretic mobility of L-glutamate oxaloacetate transaminase. Biochem. J., 81, 434-441.

3) Boyd, J.W. \& Grevilie, G.D. (1961) The intracellular distribution, latency and electrophoretic mobility of L-glutamate-oxaloacetate transaminase of rat liver. Biochem. J., 80, 18P-19P.

4) Boyde, T.R.C. \& Latner, A.L. (1962) Starch-gel electrophoresis of transaminase in human-tissue extracts and sera. Biochem. J., 82, 51P.

5) Chan, R.D., Kaplan, N.O., Levine, L. \& Zwilling, E. (1962) Nature and development of lactic dehydrogenase. Science, 136, 962-969.

6) Fleisher, G.A., Potter, C.S. \& Wakim, K.G. (1960) Separation of 2 glutamic-oxaloacetic transaminase by paper electrophoresis. Proc. Soc. Exp. Biol. Med., 103, 229-231.

7) Funao, T. \& Sugimoto, M. (1960) Enzymological studies on cardioplegia. Jap. J. legal Med., 14, 552-557. (Japanese)

8) Karmen, A., Wróblewski, F. \& LaDue, J.S. (1955) Transaminase activity in human blood. J. clin. Invest., 34, 126-131.

9) Katunuma, N., Matsuzawa, T. \& Huzino, A. (1962) Differences between the transaminases in mitochondria and soluble fraction. II Glutamic-oxaloacetic transaminase. J. Vitamin., 8, 74-79.

10) Katunuma, N., Mikumo, K., Matuda, M. \& Okada, M. (1962) Differences between the transaminases in mitochondria and soluble fraction. I Glutamic-pyruvic transaminase. J. Vitamin., 8, 68-73.

11) Lindy, S. \& Rajasalmi, M. (1966) Lactate dehydrogenase isozymes of chick embyro: Response to variations of ambient oxygen tension. Science, 153, 1401-1403.

12) Lindy, S., Turto, H.. Uitto, J., Helin, P. \& Lorenzen, I. (1972) Injury and repair in arterial tissue in the rabbit. Analysis of DNA, RNA hydroxyproline and lactate dehydrogenase in experimental arteriosclerosis. Circulat. Res., 30, 123-130.

13) Marke, Z. (1972) Morphological changes in the myocardium as substrate of "functional asymmetry" in sudden death. Forens. Sci., 1, 427-436.

14) Meyers, R.C. \& Van Remortel, H. (1968) The use of a reagent gel to locate LDH isoenzymes separated on cellulose acetate membranes. Clin. Chem., 14, 1131-1134.

15) Plagemann, P.G.W., Gregory, K.F., Swim, H.E. \& Chen, K.K.W. (1963) Canad. J. Microbiol., 9, 75. Cit. by Wilkinson, J.H., in: Isozymes, 2nd ed., Chapman and Hall Ltd., 1970, p.136.

16) Plummer, D.T., Elliott, B.A., Cooke, K.B. \& Wilkinson, J.H. (1963) Organ specificity and lactate-dehydrogenase activity. Biochem. $J ., 81,416-422$.

17) Sawaguchi, A. (1973) Studies on the activity of lactic dehydrogenase and its isozymes in acute suffocation. Forens. Sci., 2, 291-304.

18) Shaw, C.R. \& Barto, F. (1963) Genetic evidence for the subunit structure of lactate dehydrogenase isozymes. Proc. Nat. Acad. Sci. U.S., 50, 211-214.

19) Shikata, I., Maeiwa, M. \& Takehara, S. (1970) Basic Studies on the diagnosis of death from heart failure. Nature and Activity of serum transaminase (GOT) in experimental myocardosis. Jap. J. legal Med., 24, 421-426.

20) Sugai, M. (1959) A pathological study on sudden and unexpected death especially on the cardiac death autopsied by medical examiners in Tokyo. Acta Pathol. Jap., 9 (Supple.), 723-752

21) Vesell, E.S. \& Bearn, A.G. (1961) Isozymes of lactic dehydrogenase in human tissue. J. clin. Invest., 40, 586-591.

22) Wieme, R.J. \& Van Maercke, Y. (1961) The fifth (electrophoretically slowest) serum lactic dehydrogenase as an index of liver injury. Ann. N.Y. Acad. Sci., 94, 898-911.

23) Wróblewski, F. \& Gregory, K.F. (1961) Lactic dehydrogenase isozymes and their distribution in normal tissues and plasma and in disease state. Ann, N.Y. Acad. Sci., 94, 912-932. 